WESTINGHOUSE SAVANNAH RIVER COMPANY

SAVANNAH RIVER TECHNOLOGY CENTER

\title{
CLSM BLEED WATER REDUCTION TEST RESULTS (U)
}

\author{
Christine A. Langton, \\ Westinghouse Savannah River Company \\ Savannah River Technology Center, \\ Aiken, SC 29808 \\ and \\ N. Rajendran, \\ Bechtel Savannah River Company \\ Aiken, SC 29808
}

\begin{abstract}
Prepared for: Westinghouse Savannah River Company, High-Level Waste Engineering,

Savannah River Site, Aiken, SC 29808
\end{abstract}

Revision: 0

Date: April 21, 1997

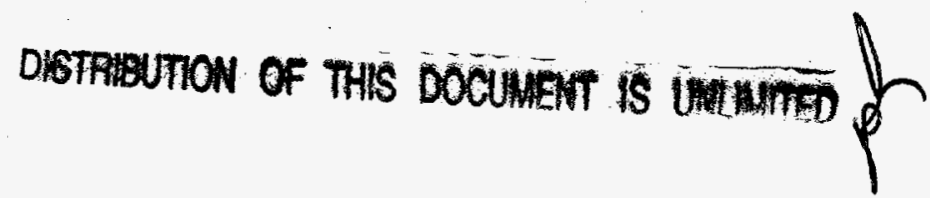




\section{DISCLAIMER}

This report was prepared as an account of work sponsored by an agency of the United States Government. Neither the United States Government nor any agency thereof, nor any of their employees, makes any warranty, express or implied, or assumes any legal liability or responsibility for the accuracy, completeness, or usefulness of any information, apparatus, product, or process disclosed, or represents that its use would not infringe privately owned rights. Reference herein to any specific commercial product, process, or service by trade name, trademark, manufacturer, or otherwise does not necessarily constitute or imply its endorsement, recommendation, or favoring by the United States Government or any agency thereof. The views and opinions of authors expressed herein do not necessarily state or reflect those of the United States Government or any agency thereof.

This report has been reproduced directly from the best available copy.

Available to DOE and DOE contractors from the Office of Scientific and Technical Information, P. O. Box 62, Oak Ridge, TN 37831; prices available from (423) 576-8401.

Available to the public from the National Technical Information Service, U. S. Department of Commerce, 5285 Port Royal Road, Springfield, VA 22161. 


\section{DISCLAIMER}

Portions of this document may be illegible electronic image products. Images are produced from the best available original document. 


\section{WESTINGHOUSE SAVANNAH RIVER COMPANY} SAVANNAH RIVER TECHNOLOGY CENTER

\section{CLSM BLEED WATER REDUCTION TEST RESULTS (U) \\ Christine A. Langton, Westinghouse Savannah River Company \\ Savannah River Technology Center, Aiken, SC 29808 \\ N. Rajendran, Bechtel Savannah River Company Aiken, SC 29808}

\section{SUMMARY}

Previous testing by BSRI/SRTC/Raytheon indicated that the CLSM specified for the Tank 20 closure generates about 6 gallons (23 liters) of bleed water per cubic yard of material $\left(0.76 \mathrm{~m}^{3}\right){ }^{1}$ This amount to about $10 \%$ of the total mixing water. HLWE requested that the CLSM mix be optimized to reduce bleed water while maintaining flow. Elimination of bleed water from the CLSM mix specified for High-Level Waste Tank Closure will result in waste minimization, time savings and cost savings.

Over thirty mixes were formulated and evaluated at the on-site Raytheon Test Laboratory. Improved low bleed water CLSM mixes were identified. Results are documented in this report.

\section{APPROACH}

The strategy for eliminating bleed water from the CLSM was to use a high range water reducing (HRWR) agent to maintain high flow with less mixing water while at the same time using another admixture to prevent the solids (sand, cement, and fly ash) from settling out of suspension. A naphalene sulfonate-formaldehyde polymer, Daracem 19, (W. R. Grace) a melamine polymer, Melment 330, (W. R. Grace) and a polycarbonated polymer, Advacast, (W. R. Grace) was the HRWR used in all of the modified mixes. Two approaches were for minimizing settling were evaluated:

1) Test air generating admixtures (foaming agents) which form bubbles that help support the solids and fluidize the slurry.

2) Test an organic polymeric thickener which is compatible with cement systems.

Two foaming admixtures, Darafill (W. R. Grace) and Rheofill (Master builders) were tested. A polymeric thickener, Kelco-Crete (Kelco/Monsanto) was also evaluated. 
WSRC-RP-97-271

April 21,1997

The following measurements were made on the modified mixes:
1) Flow
ACI 229
2) Bleed Water
ASTM C 232-92a
3) Air Content
ASTM C 23 1-91b
4) Fresh Unit Weight
ASTM C 138-92

Acceptance criteria for this series of experiments was limited to:

- $\geq \mathbf{1 0 . 5}$ inches of flow

- 0 bleed water

- $<20 \%$ air content (to minimize pumping problems per W. R. Grace recommendation)

\section{RESULTS AND DISCUSSION}

Mix designs and results are summarized in Table 1. The cement contents ranged from 100 to 150 $\mathrm{lbs} / \mathrm{yd}^{3}$. Fly ash contents ranged from 300 to and $500 \mathrm{lbs} / \mathrm{yd}^{3}$. Sand contents ranged from 2150 to $2300 \mathrm{lbs} / \mathrm{yd}^{3}$. The types and amounts of admixtures and the amount of water were varied.

Initial results indicated:

- Reducing mixing water reduces both bleed water and flow.

- Reducing mixing water and adding a HRWR reduces bleed water without reducing flow. However this did not eliminate all of the bleed water.

- Beyond a certain amount, further addition of HRWR gives no additional improvement in bleed water reduction. Instead it segregates from the CLSM (rises to the surface) as bleed HRWR.

- No bleed water was obtained from CLSM mixes with a high amount of foaming admixture. However, these mixes did not achieve the flow requirement of $>10.5$ inches. These mixes had flows of less than 9 inches.

- Darafill (W. R. Grace) gave better results than Rheofill (Master builders) all other parameters held constant.

- Darafill is a liquid. Rheofill is a powder. The powder is easier to incorporate in the mix than the liquid.

- The unit weight for the mixes containing the foaming agents is 10 to $15 \%$ less than the original reference CLSM mix.

- CLSM mixes with foaming agents display very little segregation and are more cohesive than the reference mix.

- Mixes containing Kelco-Crete visually appear very cohesive and rather "stiff." However, these mixes are very flowable when subjected to the CLSM flow test.

- Kelco-Crete is available in powder form or as a dispersion in a liquid HRWR. The powdered form was used in these tests. The powder was mixed with a portion of the fly ash and added at the end of the mixing cycle to achieve maximum effect.

- The Kelco-Crete mixes have unit weights of greater than $130 \mathrm{lbs} / \mathrm{ft}^{3}$ (comparable to pumpable concrete) and are expected to be pumpable.

- Increasing the water and/or the HRWR in the Kelco-Crete mixes increased the flow.

- Increasing the HRWR and decreasing the mixing water, decreased the bleed water in the KelcoCrete mixes.

- Increasing the Kelco-Crete, all other parameters held constant, decreased the flow but also decreased the bleed water.

- Set times of the CLSM mixes were affected by the specific admixtures tested.

- Other modifications, such as, substituting Type III cement for Type I cement in the CLSM mix design did not result in any improvement with respect to bleed water reduction. 


\section{CONCLUSIONS}

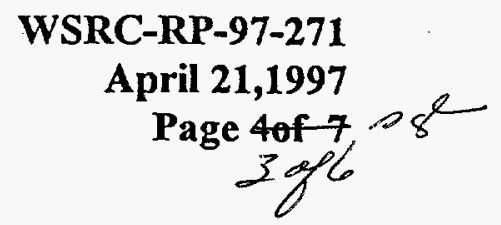

Improved low bleed water mixes were developed as the result of this work. The ingredients and proportions for 2 promising mix designs are listed in Table 2 along with the original CLSM mix specified for High Level Waste Tank Closure.

Two different systems for reducing bleed water were identified. The differences in these systems can be attributed to the very different ways that foaming agent and thickening agent work to prevent settling. The foaming agents add air bubbles to the mix which help to fluidize the slurry and to suspend the solids. This results in flowable light weight mixes with good cohesion and mix uniformity. The thickening admixture adds a polymer structure to the mix which also enhances flow and cohesion while maintaining a unit weight similar to that of pumpable concrete. Selection of the system best suited for a backfill will depend on the specific engineering requirements of that application.

Two mixes one from each system are listed in Table 2. Field mixing and pumping tests were conducted to confirm the laboratory results and to finalize a mix design for High-Level Waste Tank Closure. Mixing was conducted in a Ready Mix truck. Mix $Q$ was more difficult to pump than Mix TW-10. Mix $Q$ also showed some sand segregation as a result of pumping.

\section{RECOMMENDATIONS.}

Based on the laboratory and field batching and pumping tests, Mix TW-10 is recommended for Tank Closure Specification, C-SPP-F-00038. A DCF must be submitted and approved.

Field mixing and pumping must be evaluated for specific equipment supplied by Throop and BSRI at the F-Area portable batching plant.

\section{REFERENCES}

WSRC-RP-96-554, N. Rajendran, C. Langton and T. M. Nixon, Bleed Water Testing Program for Controlled Low Strength Material (CLSM) (U), 10/22/96. 
TABLE 1 - Modified CLSM Mix Designs and Test Results for Bleed Water Reduction Program

\begin{tabular}{|c|c|c|c|c|c|c|c|c|c|c|c|c|c|}
\hline $\begin{array}{l}\text { Mix } \\
\#\end{array}$ & $\begin{array}{l}\text { Cement } \\
\text { Type I } \\
\text { lbs/cyd }\end{array}$ & $\begin{array}{l}\text { Fly ash } \\
\text { Class F } \\
\text { lbs/cyd }\end{array}$ & $\begin{array}{l}\text { Sand } \\
\text { C.33 } \\
\text { lbs/cyd }\end{array}$ & $\begin{array}{l}\text { Water } \\
\text { galeyd }\end{array}$ & $\begin{array}{l}\text { HRWR } \\
\text { oz/cyd }\end{array}$ & $\begin{array}{l}\text { Darafill } \\
\text { oz/cyd }\end{array}$ & $\begin{array}{l}\text { Rheofill } \\
\text { gms/cyd }\end{array}$ & $\begin{array}{l}\text { Kelco-Crete } \\
\text { gms/cyd }\end{array}$ & $\begin{array}{c}\text { Flow (Ave.) } \\
\text { inch } \\
\end{array}$ & $\begin{array}{l}\text { Bleed } \\
\text { Water } \\
\text { vol. \% }\end{array}$ & $\underset{\text { Content } \%}{\text { Air }}$ & $\begin{array}{l}\text { Fresh } \\
\text { Unit Wt } \\
\text { lbo/cuft }\end{array}$ & $\begin{array}{l}\text { Compressive } \\
\text { Strength Psi }\end{array}$ \\
\hline $\bar{C}$ & 100 & 300 & 2230 & 50.8 & 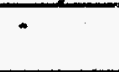 & 2.0 & - & $\overline{-}$ & 10.4 & $\begin{array}{c}7.5 \\
(24 \mathrm{hr})\end{array}$ & 20.5 & 105.3 & $45(28 d)$ \\
\hline $\bar{D}$ & 100 & 400 & 2300 & 40.9 & - & 2.5 & 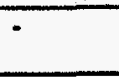 & - & 10.4 & $\begin{array}{c}5.1 \\
(24 \mathrm{hr})\end{array}$ & 17.0 & 107.7 & \\
\hline $\bar{E}$ & 100 & 400 & 2200 & 50.0 & 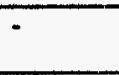 & 2.5 & - & - & 10.5 & $\begin{array}{c}5.7 \\
(24 \mathrm{hr})\end{array}$ & 20.0 & 107.2 & $45(28 d)$ \\
\hline $\bar{F}$ & 100 & 450 & 2150 & 49.23 & - & 3.0 & - & - & 10.0 & $\begin{array}{c}4.2 \\
(24 \mathrm{hr})\end{array}$ & 19.0 & 109.9 & \\
\hline $\bar{G}$ & 100 & 450 & 2100 & 51.7 & $\cdot$ & 3.5 & - & - & 10.5 & $\begin{array}{c}6.3 \\
(24 \mathrm{hr})\end{array}$ & 17.0 & 102 & \\
\hline $\mathrm{H}$ & $100^{*}$ & 400 & 2150 & 52.5 & 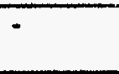 & 3.5 & - & - & 11.5 & $\begin{array}{c}4.5 \\
(24 \mathrm{hr})\end{array}$ & 14.0 & 113.2 & \\
\hline I & 100 & 400 & 2150 & 48.2 & - & 3.5 & $\cdot$ & $\cdot$ & 11.1 & $\begin{array}{c}3.5 \\
(24 \mathrm{hr})\end{array}$ & 19.5 & 106.4 & \\
\hline J & 100 & 400 & 2250 & 49.9 & - & 3.5 & - & 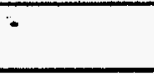 & 10.5 & $\begin{array}{c}3.3 \\
(24 \mathrm{hr}) \\
\end{array}$ & 17.0 & 112.9 & \\
\hline $\bar{K}$ & 100 & 400 & 2150 & 44.0 & $40+$ & 3.5 & - & - & 10.3 & $>10$ & 20.0 & 105.6 & \\
\hline $\mathrm{L}$ & 100 & 400 & 2250 & 42.5 & $60+$ & 3.5 & $\div$ & - & 10.8 & $>10$ & 19.0 & 109.1 & \\
\hline $\mathrm{P}$ & 100 & 400 & 2200 & 47.5 & $45+$ & 4.0 & - & - & 10.5 & $>10$ & 18.5 & 110.7 & \\
\hline Q & 100 & 100 & 150 & 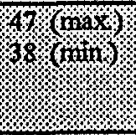 & $45 \%$ & 16 & $\%$ & 8 & $1 \%$ & 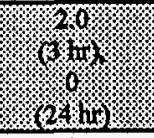 & 208 & $106 \%$ & $40 \%$ \\
\hline$Q-2$ & 100 & 400 & 2150 & 36.8 & $45+$ & 4.5 & - & $\cdot$ & 9.0 & 0 & 23.0 & 103.0 & $\begin{array}{c}50(7 d) \\
56(14 d) \\
\end{array}$ \\
\hline $\bar{R}$ & 100 & 400 & 2150 & 52.8 & $\cdot$ & $\cdot$ & 80 & $\cdot$ & 11.8 & $\begin{array}{c}6.4 \\
(24 \mathrm{hr})\end{array}$ & 23.0 & 96.0 & \\
\hline$S$ & 100 & 400 & 2150 & 45.0 & $45+$ & $\cdot$ & 100 & $\cdot$ & 11.5 & $\begin{array}{c}5.5 \\
(24 \mathrm{hr})\end{array}$ & 25.0 & 102.0 & \\
\hline $\bar{T}$ & 100 & 300 & 2300 & 43.8 & $45+$ & $\cdot$ & 100 & $\cdot$ & 11.5 & $\begin{array}{c}6.0 \\
(24 \mathrm{hr})\end{array}$ & 20.0 & 106.2 & \\
\hline $\mathrm{V}$ & 100 & 400 & 2150 & 57.0 & 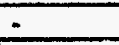 & - & - & 267.6 & 11.0 & $>10$ & 3.0 & 131.2 & \\
\hline$A$ & 100 & 400 & 2150 & 46.4 & $135.3+$ & $\cdots$ & $=$ & 340.0 & 11.5 & $>10$ & 3.2 & 130.3 & \\
\hline$A=1$ & 150 & 500 & 2300 & 45.6 & $101.4+$ & $=$ & - & 227.0 & 8.4 & $0.4(24 \mathrm{hr})$ & 2.8 & 133.5 & \\
\hline A-2 & 150 & 500 & 2300 & 51.3 & $101.4+$ & - & - & 227.0 & 12.0 & $0.6(24 \mathrm{hr})$ & 1.8 & 132.8 & \\
\hline$A-3$ & 150 & 500 & 2300 & 45.6 & $101.4+$ & $\div$ & - & 227.0 & 8.3 & $0.5(24 \mathrm{hr})$ & 5.5 & 129.7 & \\
\hline$A=10$ & 150 & 500 & 2300 & 52.2 & $135.3+$ & - & $\div$ & 341.0 & 10.5 & $0 \quad(4 d)$ & 3.5 & 136.8 & \\
\hline$A-4$ & 150 & 500 & 2300 & 51.3 & $169.1+$ & - & $\therefore$ & 227.0 & 10.5 & $2.8(4 d)$ & 3.2 & 131.0 & \\
\hline
\end{tabular}

- Type III Cement used

Highlighted mixes gave promising results

$\sim$ Percent bleed water is calculated as follows: volume of mixing water divided by volume of the bleed water times 100 .

+ Daracem 19 
TABLE 1 Continued.

\begin{tabular}{|c|c|c|c|c|c|c|c|c|c|c|c|c|c|c|}
\hline $\begin{array}{l}\text { Mix } \\
\#\end{array}$ & $\begin{array}{l}\text { Cement } \\
\text { Type I } \\
\text { lbs/cyd }\end{array}$ & $\begin{array}{l}\text { Fly ash } \\
\text { Class F } \\
\text { lbs/cyd }\end{array}$ & $\begin{array}{l}\text { Sand } \\
\text { C-33 } \\
\text { lbs/cyd }\end{array}$ & $\begin{array}{l}\text { Water } \\
\text { galcyd }\end{array}$ & $\begin{array}{l}\text { HRWR } \\
\text { oz/cyd }\end{array}$ & $\begin{array}{l}\text { Darafill } \\
\text { oz/cyd }\end{array}$ & $\begin{array}{l}\text { Rheofill } \\
\text { gms/cyd }\end{array}$ & $\begin{array}{l}\text { Other } \\
\text { Admixture } \\
\text { lbs/cyd }\end{array}$ & $\begin{array}{l}\text { Kelco-Crete } \\
\text { lot 49917K } \\
\text { gms/cyd }\end{array}$ & $\begin{array}{c}\text { Flow (Ave.) } \\
\text { inch }\end{array}$ & $\begin{array}{l}\text { Bleed } \\
\text { Watem } \\
\text { vol. } \%\end{array}$ & $\begin{array}{c}\text { Air } \\
\text { Content } \\
\%\end{array}$ & $\begin{array}{l}\text { Fresh } \\
\text { Unit Wt } \\
\text { lbs/cuft }\end{array}$ & $\begin{array}{l}\text { Compressive } \\
\text { Strength, Psi }\end{array}$ \\
\hline TW-1 & 150 & 500 & 2300 & 55.1 & $73.5+$ & - & $\cdot$ & $6.5 \mathrm{Ca}(\mathrm{OH})_{2}$ & 341 & 10.8 & $\begin{array}{l}0(2 \mathrm{hr}) \\
0(15 \mathrm{hr})\end{array}$ & $\cdot$ & - & $100(7 \mathrm{~d})$ \\
\hline TW.2 & 150 & 500 & 2300 & 53.9 & $73.5+$ & - & $\cdot$ & $4.9 \mathrm{Ca}(\mathrm{OH})_{2}$ & 300 & 11 & $\begin{array}{l}<2 \%(2 \mathrm{hr}) \\
0(15 \mathrm{hr})\end{array}$ & - & 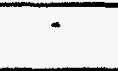 & $90(7 \mathrm{~d})$ \\
\hline TW-3 & 150 & 500 & 2300 & 62.9 & $77.6++$ & - & - & - & 341 & 11 & $\begin{array}{l}<1 \%(2 \mathrm{hr}) \\
0(15 \mathrm{hr})\end{array}$ & & & $95(7 d)$ \\
\hline TW 4. & 150 & 500 & 2300 & 62.9 & $75+4$ & $=$ & 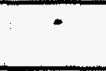 & 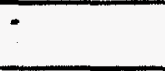 & 341 & 10.5 & $\begin{array}{l}0(2 \mathrm{hr}) \\
0(15 \mathrm{hr})\end{array}$ & - & - & $82(7 d)$ \\
\hline TW.5 & 150 & 500 & 2300 & 62.9 & $135 * *$ & - & - & - & 341 & 10.6 & $\begin{array}{l}0(2 \mathrm{hr}) \\
0(15 \mathrm{hr})\end{array}$ & - & - & $118(7 d)$ \\
\hline TW-6 & 150 & 500 & 2300 & 62.9 & $75+t$ & - & - & $\cdot$ & $275^{n}$ & 11.25 & $\begin{array}{l}<2 \%(2 \mathrm{hr}) \\
0(15 \mathrm{hr})\end{array}$ & - & - & $85(7 d)$ \\
\hline TW-7 & 150 & 500 & 2300 & 63 & $75+t$ & - & - & $3\left(\mathrm{CaNO}_{3}\right)$ & $275^{\circ}$ & 11.25 & $\begin{array}{l}<1 \%(2 \mathrm{hr}) \\
<1 \%(15 \mathrm{hr}) \\
0(36 \mathrm{hr})\end{array}$ & - & - & $86(7 d)$ \\
\hline TW-8 & 200 & 450 & 2300 & 65.5 & $75+4$ & - & - & - & $275^{\prime}$ & 11 & $\begin{array}{l}<2 \%(2 \mathrm{hr}) \\
<2 \%(15 \mathrm{hr}) \\
0(36 \mathrm{hr})\end{array}$ & - & - & $118(7)$ \\
\hline TW-9 & 150 & 500 & 2300 & 65.5 & $75++$ & - & $\cdot$ & - & $275^{1}$ & $\begin{array}{l}11 \\
10.5,5 \mathrm{~min} \\
\text { rest } \\
8.4,17 \mathrm{~min} \\
\text { rest } \\
9.5, \text { remix }\end{array}$ & $\begin{array}{l}3 \%(2 \mathrm{hr}) \\
2 \%(15 \mathrm{hr}) \\
0(36 \mathrm{hr})\end{array}$ & 7.8 & 124 & $158(7 d)$ \\
\hline $10 \times 10$ & 150 & 800 & 6300 & 63 & \%. & 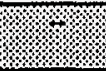 & $\%$ & & $6 \%$ & \% $18 \%$ & 6) & 8 & 123. & $18 \%$ \\
\hline
\end{tabular}

* Type III Cement used

Highlighted mixes gave promising results

$\sim$ Percent bleed water is calculated as follows: volume of mixing water divided by volume of the bleed water times 100 .

+ Daracem 19

+ Adva Cast

** Melment 33 
TABLE 2 - Summary of Improved CLSM Mix Designs and Reference Tank Closure Mix

\begin{tabular}{|c|c|c|c|c|c|c|c|c|}
\hline MIX\# & $\begin{array}{l}\text { Cement } \\
\text { Type I } \\
\text { lbs/cyd }\end{array}$ & $\begin{array}{l}\text { Fly ash } \\
\text { Class F } \\
\text { lbs/cyd }\end{array}$ & $\begin{array}{c}\text { Sand } \\
\text { C-33 } \\
\text { lbs/cyd }\end{array}$ & $\begin{array}{c}\text { Water } \\
\text { gals/cyd }\end{array}$ & $\begin{array}{l}\text { HRWR } \\
\text { oz/cyd }\end{array}$ & $\begin{array}{l}\text { Darafill } \\
\text { oz/cyd }\end{array}$ & $\begin{array}{l}\text { Kelco- } \\
\text { Crete } \\
\text { gms/cyd }\end{array}$ & Remarks \\
\hline $\mathbf{Q}$ & 100 & 400 & 2150 & $\begin{array}{l}47 \text { (mas.) } \\
38 \text { (min.) }\end{array}$ & $45^{*}$ & 4.5 & - & $\begin{array}{l}\text { Excellent } \\
\text { flow, } \\
\text { Zero } \\
\text { bleed } \\
\text { after } 24 \\
\text { hours } \\
\end{array}$ \\
\hline TW-10 & 150 & 500 & 2300 & $\begin{array}{l}63(\max )) \\
60(\min )\end{array}$ & $\begin{array}{c}75^{* *} \\
90^{* *} \\
(\max .)\end{array}$ & 0 & 275 & \begin{tabular}{|l} 
Excellent \\
flow, \\
Zero \\
bleed \\
after 15 \\
hours \\
\end{tabular} \\
\hline $\begin{array}{c}\text { Ref. } \\
\text { Mix } \\
\text { OPAE } \\
\text { XE-X- } \\
\text { P-O-X }\end{array}$ & 150 & 500 & 2515 & 66.0 & - & - & - & $\begin{array}{l}\text { Excellent } \\
\text { flow, } \\
10 \% \\
\text { bleed } \\
\text { water } \\
\text { after } 24 \\
\text { hours }\end{array}$ \\
\hline
\end{tabular}

* Daracem 19 (W. R. Grace)

** Advacast or Advaflow (W. R. Grace) 